\title{
What's New In Asthma Management
}

\author{
Dr Grace Chiang
}

\section{SFP202 I; 47(7)}

Asthma is a major non communicable disease. It is the most common chronic respiratory illness worldwide, affecting both children and adults. ${ }^{1,2}$ In 2019, asthma affected an estimated 262 million people and caused 461000 deaths. ${ }^{1,2}$ While asthma is incurable, those with asthma can enjoy a normal and active lifestyle. Asthma can be controlled with holistic management including non-pharmacological interventions such as healthy lifestyle choices and pharmacological interventions with inhaled medications. ${ }^{3}$ Asthma management makes up a large part of primary care practice and family physicians need to be well-versed in asthma diagnosis and management. This issue will provide an update on the latest evidence-based treatment options in asthma management. The College is pleased to partner with AstraZenaca to conduct this Family Practice Skills Course for our doctors.

In Unit 1, Dr Adrian Chan writes about the current guideline recommendations and evidence-based treatment for the management of asthma in 2021. Dr Chan also highlighted the problems associated with short-acting beta-agonists (SABA) monotherapy and the optimal use of inhaled corticosteriods (ICS) as part of long -term asthma management.

In Unit 2, Dr Akash Verma writes about the management of incidental lung nodules. Dr Verma highlights the importance of early investigation when lung nodules are detected as diagnosis of cancer in the early-stage influences survival. Dr Verma also discusses why lung nodules in young women and non-smokers should not be assumed to be benign.

In Unit 3, Dr Adrian Chan discusses the simple decisions and diagnostic steps that may aid general practitioners in identifying patients who might benefit from a referral to tertiary care. Dr Chan also briefly discusses the impact of oral corticosteroids (OCS) use and the challenges of the COVID-19 lockdown on asthma management.

In this issue, A/Prof Goh Lee Gan has also selected ten current readings on topics related to contemporary asthma management and management of lung nodule findings. These readings include articles on the characterization and management of severe asthma, insights on primary care asthma management, patient's experiences on asthma management, and the management of lung nodules found on imaging.

In this issue, we also have 3 original articles, one PRISM article, and one case report. The first original article is by Dr Jared Ng, A/Profs Teo Choon Liang and Goh Lee Gan. This article summarizes the role of primary care physicians in the promotion of mental health and discusses the primary, secondary and tertiary prevention of mental illnesses. The second original article is by Dr Loh Jiashen and Dr Andrew Peh. This article provides an overview of the COVID-19 vaccination landscape, reviews important concepts regarding COVID-19 vaccination, describes important vaccine reactions and assesses their potential implications towards achieving herd immunity. The third original article is by Dr Vikneswaran Namasivayam and this article reviews the management of Helicobacter pylori in gastric cancer prevention and the developments in investigation and management of early gastric cancer. The PRISM article is by Dr Chong Yong He and A/Prof Goh Lee Gan who describe a case of Attention Deficit Hyperactivity Disorder (ADHD) in a young boy and explores how this case was managed; this article also discusses the diagnosis and treatment of ADHD and the role family physicians play in managing ADHD. The case report is by Dr Wong Ann Mei who describes a gentleman with high fall risks and how prevention of falls was achieved.

\section{REFERENCES}

I. Vos T, Lim SS, Abbafati C, Abbas KM, Abbasi M, Abbasifard M, Abbasi-Kangevari M, Abbastabar H, Abd-Allah F, Abdelalim A, Abdollahi M. Global burden of 369 diseases and injuries in 204 countries and territories, 1990-2019: a systematic analysis for the Global Burden of Disease Study 2019. The Lancet. 2020 Oct 17;396(I0258): 1204-22.

2. World Health Organization, 2020. Assessing national capacity for the prevention and control of noncommunicable diseases: report of the 2019 global survey.

3. Cloutier MM, Baptist AP, Blake KV, Brooks EG, Bryant-Stephens T, DiMango E, Dixon AE, Elward KS, Hartert T, Krishnan JA, Ouellette DR. 2020 focused updates to the asthma management guidelines: a report from the National Asthma Education and Prevention Program Coordinating Committee Expert Panel Working Group. Journal of Allergy and Clinical Immunology. 2020 Dec I;| $146(6): 1217-70$. 